\title{
Psychoacoustic analysis of contra-rotating propeller noise for Unmanned Aerial Vehicles
}

\author{
Antonio J. Torija, ${ }^{1 \text { a }}$ Paruchuri Chaitanya, ${ }^{2,}$ and Zhengguang $\mathrm{Li}^{3}$ \\ ${ }^{1}$ Acoustics Research Centre, University of Salford, Manchester, M5 4WT, United Kingdom \\ ${ }^{2}$ Institute of Sound and Vibration Research, University of Southampton, Southampton, SO17 1BJ, United \\ Kingdom \\ ${ }^{3}$ Department of Architecture, Zhejiang University of Science and Technology, Hangzhou, 310023, P.R. China
}

Unmanned aerial vehicle (UAV) technologies are rapidly advancing due to the unlimited number of applications from parcel delivery to people transportation. As the UAV market expands, community noise impact will become a significant problem for public acceptance. Compact drone architectures based on contra-rotating propellers bring significant benefits in terms of aerodynamic performance and redundancy to ensure vehicle control in case of component failure. However, contra-rotating propellers are severely noisy if not designed appropriately. In the framework of a perception-influenced design approach, this paper investigates the optimal rotor spacing distance configuration to minimise noise annoyance. On the basis of a series of psychoacoustic metrics (i.e. loudness, fluctuation strength, roughness, sharpness and tonality) and psychoacoustic annoyance models, the optimal rotor axial separation distance (expressed as a function of propeller blade diameter) is at a range of 0.2 to 0.4 . This paper also discusses the performance of currently available psychoacoustic models to predict propeller noise annoyance, and defines further work to develop a psychoacoustic annoyance model optimised for rotating systems.

a A.J.TorijaMartinez@,salford.ac.uk 


\section{I. INTRODUCTION}

2 New aviation markets, such as Urban Air Mobility (UAM) operations for passengers and drone

3 operations for goods' deliveries and blue light services, are estimated to have a global potential of

4 between $\$ 132$ and $\$ 227$ billion over the next 20 years (ATI, 2019). As the drone delivery market

5 intensifies over the coming years, the payload requirement is predicted to increase by a factor of 50

6 to 100, leading to further problems with their public acceptance; with noise becoming a primary

7 focus. This increase in payload requirements can only be achieved with compact drone architectures

8 such as co-axial or overlapping propellers. The use of contra-rotating propellers in Unmanned

9 Aerial Vehicles (UAVs) has the benefit of increasing aerodynamic performance (Stract et al., 1981),

10 reducing the UAV's plan size and adding redundancy in case of component failure (McKay et al.,

11 2019).

12 However, the small tip-to-tip spacing between contra-rotating propellers results in a significant

13 source of noise due to blade interaction effects (Tinney and Sirohi, 2018; Alexander et al., 2019).

14 Extensive laboratory testing has found that in the frequency spectra of multi-rotor UAV there are

15 significant sound levels at higher harmonics of the blade passage frequency, which seems to be

16 caused by interaction noise from disturbed inflow due to other rotor blades or the fuselage

17 (Magliozzi, 1991; Cabell et al., 2016; Torija et al., 2019). In an experimental investigation of static

18 multi-rotor contra-rotating UAV propellers, McKay et al. (2019) observed that potential field

19 interaction tones are about $20 \mathrm{~dB}$ higher than rotor alone tones at typical ground observer locations

20 with a hovering UAV. This suggests that proper design of multi-rotor contra-rotating UAV

21 propellers to minimise interaction between rotors can lead to significant reductions in noise

22 emission.

23 The noise sources on a co-axial propeller system can be categorized into either rotor self-noise

24 or interaction noise. Rotor self-noise is principally composed of tonal components and has 
25 contributions due to the steady loading and aerofoil thickness, while the broadband component is

26 relatively weak (Marte and Kurtz, 1970). An interaction source is generated when the spiraling wake

27 and tip vortex from the upper propeller interacts with the lower propeller. At sufficiently small rotor

28 separation distances, an additional interaction noise source is present arising from the interaction of

29 the potential near field of each propeller with the other (Heff, 1990). A more recent study by

30 Chaitanya et al. (2020) performed a detailed investigation on the sensitivity of the aerodynamic and

31 aeroacoustic performance to the axial separation distance between a counter-rotating propeller

32 configuration. An optimum separation distance to diameter ratio for maximum efficiency and

33 minimum radiated noise was found to be at 0.25 based on overall sound power level. The reason

34 behind this optimum is attributed to the balance between potential field interactions and tip-vortex

35 interactions radiated from the contra-rotating configuration. The current paper extends their work

36 to perform psychoacoustic optimization of contra-rotating propellers.

37 Anghinolfi et al. (2016) carried out a psychoacoustic optimization of blade spacing in subsonic,

38 open, or nearly open axial-flow rotors. This optimization focused only on tonal noise and the

39 objective function was based on the Tone-to-Noise Ratio (TNR) metric. They found optimal blade

40 spacing for different numbers of blade rotors as a function of TNR and level of the highest tonal

41 peak. However, these results do not have direct relation to loudness or other psychoacoustic

42 features.

43 The perception-influenced design approach (Rizzi, 2016) aims to incorporate human response

44 into the process of creating low-noise aircraft. Metrics that correlate well with human response to 45 noise can potentially be incorporated into the aircraft design cycle to effectively reduce community

46 noise impact (Krishnamurthy et al., 2018). Current noise certification metrics do not necessarily

47 reflect the characteristics of noise signatures of unconventional aircraft designs (Rizzi, 2016;

48 Christian and Cabell, 2017; Torija et al., 2019), and therefore may not be able to predict human 
49 response. Torija et al. (2019) found that the Effective Perceived Noise Level (EPNL) is unable to

50 account for the perceptual effect of series of complex tones spaced evenly across the frequency

51 spectrum with relatively even sound levels, which is typical of multi-rotor vehicles (Cabell et al.,

52 2016; Torija et al., 2019). Other metrics, such as the Sound Exposure Level (SEL), do not account

53 for the effects of tonal noise, which is a major contributor towards the perceived annoyance due to

54 aircraft noise (Angerer et al., 1991; Berckmans et al., 2008; More, 2011; White et al., 2017).

55 Therefore, the use of current noise certification metrics for aircraft design might lead to suboptimal

56 solutions.

57 Psychoacoustic metrics have been widely applied to improve the sound quality of different

58 consumer products, especially in the automotive industry (Lyon, 2003). Psychoacoustic metrics,

59 such as loudness, sharpness, tonality, roughness and fluctuation strength, are good indicators of how

60 the human auditory system reacts to different features of acoustic stimuli (Zwicker and Fastl, 1999).

61 Loudness measures the sensation of sound intensity. Sharpness and tonality describe the perceptual

62 effects of spectral imbalance of the sound towards the high frequency region, and the presence of

63 spectral irregularities or tones respectively. Fluctuation strength and roughness describe how slow

64 and rapid fluctuations, respectively, of the sound level are perceived. The psychoacoustic metrics

65 sharpness, tonality and fluctuation strength have been suggested as good indicators of rotorcraft

66 noise annoyance (Krishnamurthy et al., 2018; Boucher et al., 2020). Investigating the performance

67 of different psychoacoustic metrics to account for the perception of different aspects of aircraft

68 noise, Barbot et al. (2008) found fluctuation strength as a good indicator of perceptual effects of

69 turbulence and sharpness as a good indicator of the perceptual effects of high frequency noise.

70 Torija et al. (2019) found that Aures/Terhardt tonality (Aures, 1985b) improves on the EPNL Tone

71 Correction in terms of accounting for the presence of complex tones in aircraft noise. 
72 Perception of mechanical sounds is a complex process due to the amount of noise features

73 involved (e.g. tonal components, amplitude modulated sounds, etc.). To address this issue, Zwicker

74 and Fastl (1999) proposed a model for combining several psychoacoustic metrics into one model to

75 quantify annoyance (hereinafter called Zwicker's model for short). Using the Zwicker's

76 psychoacoustic annoyance (PA) model, relative annoyance degrees of different noise samples can be

77 estimated from measures of loudness, sharpness, fluctuation strength and roughness. However,

78 Zwicker's PA model does not include a factor accounting for the influence of the tonality on noise

79 annoyance. To improve accuracy in the estimation of relative annoyance degrees caused by several

80 types of tonal/atonal noises, Di et al. (2016) carried out an update of Zwicker's PA model aiming at

81 tonal noises. More (2011) developed a modified version of Zwicker's PA model based on the results

82 of seven psychoacoustic tests for several aircraft sounds with varying psychoacoustic parameters.

83 The modified PA model developed by More, which includes a term based on Aures/Terhardt

84 tonality and loudness to account for the perceptual effect of tonal noise, was found able to

85 accurately predict aircraft noise annoyance.

86 The aim of this paper is to perform a psychoacoustic analysis of a single static contra-rotating

87 propeller mounted in an anechoic chamber. A set of psychoacoustic metrics are calculated for a

88 series of far-field microphone measurements with different separation distance between the contra-

89 rotating propellers. The contribution of each noise source component on the co-axial propeller

90 under study is evaluated from a perceptual standpoint, using relevant psychoacoustic metrics.

91 Working towards the development of a framework for the psychoacoustic optimisation of novel

92 aerial vehicles, this paper investigates the optimal distance between contra-rotating propellers to

93 minimise psychoacoustic impact. The performance of PA models to predict noise annoyance for

94 propeller systems is evaluated and discussed. The main assumption in this paper is that PA models 
95 optimized for propeller noise annoyance can be used to inform propeller design for lower 96 psychoacoustic impact.

97 This paper is structured as follows: Section II describes the experimental setup for acoustic 98 measurements and the metrics for psychoacoustic analysis; Section III presents and discusses the 99 experimental results and are followed by the main conclusions of this work in section IV.

100

\section{EXPERIMENTAL AND PSYCHOACOUSTIC METHODS}

\section{A. Experimental set-up and procedure}

The overlapping rotor test rig designed and manufactured at the University of Southampton consisted of two FOXTECH W61-35 brushless DC (BLDC) (16 poles) 700W motors mounted on a carbon fibre beam as shown in Fig. 1. A commercially available T-Motor 16 inch 5.4 inch rotor was used for this overlapping rotor propulsion system analysis. Two Hyperion HP-EM2-TACHBL sensors were used to measure the precise Rotations Per Minute (RPM). Two Maytech 40A-OPTO speed controllers were used to accurately control the BLDC motors. The overlapping rig allowed manipulation of the propulsion system in both rotor horizontal separation distance d/D (with D as the rotor diameter) and rotor axial separation distance $z / D . z / D$ rotor separation was achieved by a custom linear actuator traversing the upper rotor. All of the tests for this study were achieved when the lower rotor plane was at least three rotor diameters away from the ground with anechoic wedges beneath. The selected lead screw and stepper motor configuration allows for $z / D$ variations varying of 0.05 to 1 . Sixteen $z / D$ positions were tested: $0.05,0.075,0.1,0.125,0.15,0.175,0.2,0.25,0.3$, $0.35,0.4,0.45,0.5,0.6,0.8$ and 1 . The combined thrust of the dual-rotor propulsion system is varied from 2 to $20 \mathrm{~N}$ in steps of $2 \mathrm{~N}$. Although 10 thrust settings were measured, the results shown in this paper refer to a thrust of $10 \mathrm{~N}$ (varying thrusts lead to changes in magnitudes, but do not alter the trends shown below). A detailed description of the rig is presented by Brazinskas (2019). 


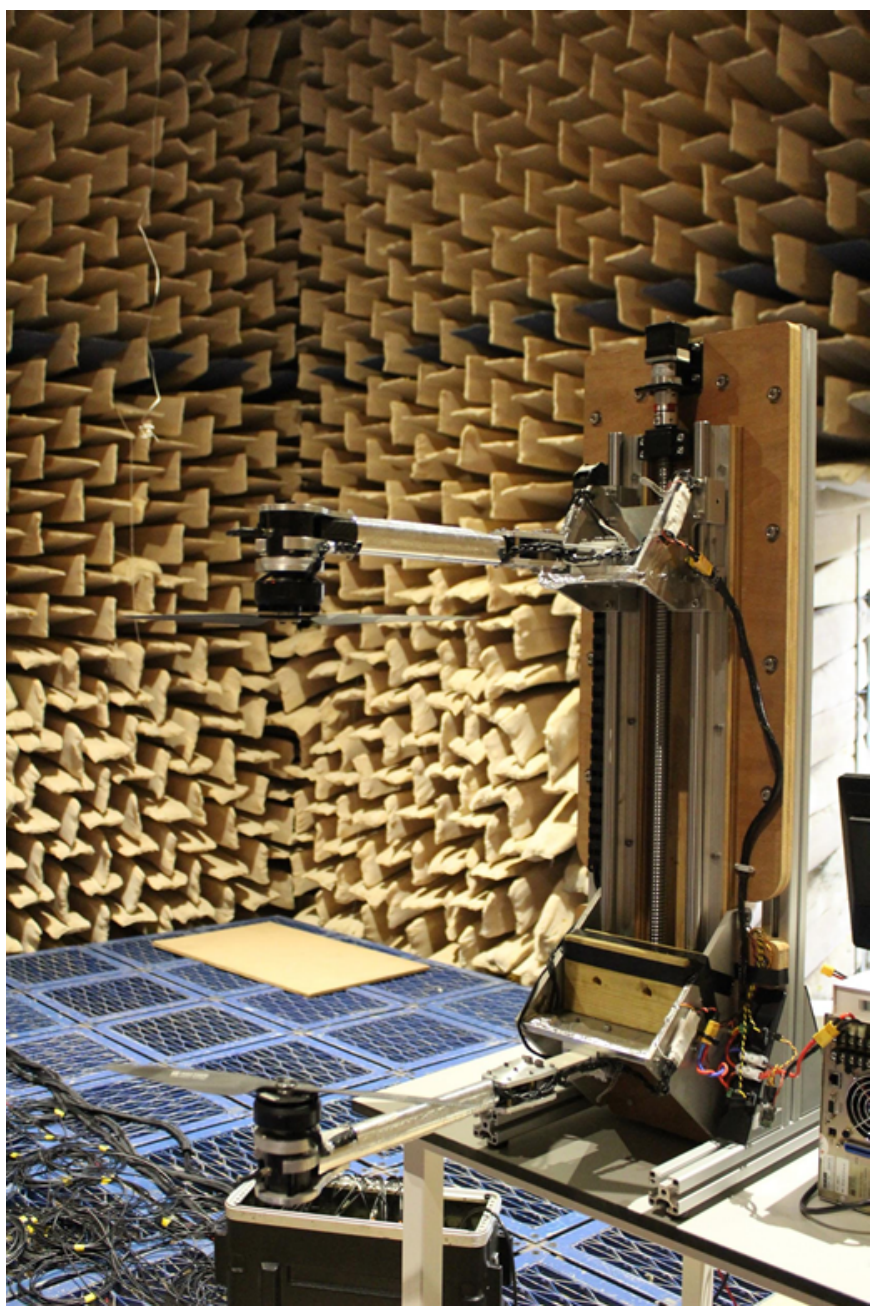

119 FIG. 1. (Color online). Photograph of overlapping propeller rig within the anechoic chamber of the

120 Institute of Sound and Vibration Research at the University of Southampton.

\section{B. Far-field noise measurements}

The overlapping far-field noise measurements were carried out at the Institute of Sound and

123 Vibration Research's open-jet wind tunnel facility. The overlapping rotor test rig was located within

124 an anechoic chamber, of dimension $8 \mathrm{~m} \times 8 \mathrm{~m} \times 8 \mathrm{~m}$ as shown in Fig. 1. The walls, acoustically

125 treated with glass wool wedges, allow a cut-off frequency of $80 \mathrm{~Hz}$.

126 Far-field noise measurements were made using 10, 1/2 in. condenser microphones (B\&K type 127 4189) located at a constant radial distance of $2.5 \mathrm{~m}$ from the centre of the propellers. These 128 microphones were placed at emission angles of between 12 and 102 degrees measured relative to the 
rotor axis. Measurements were carried out for $10 \mathrm{~s}$ duration at a sampling frequency of $50 \mathrm{kHz}$, and the noise spectra was calculated with a window size of 1024 data points corresponding to a

131 frequency resolution of $48.83 \mathrm{~Hz}$ and a Bandwidth-Time (BT) product of about 500, which is

132 sufficient to ensure negligible variance in the spectral estimated at this frequency resolution. Please

133 note that the data analysed in this paper is same as the data presented in Chaitanya et al. (2020).

\section{Psychoacoustic data analysis}

Unlike physical quantities (e.g. sound pressure level), psychoacoustic metrics provide a linear representation of human hearing perception (HEAD Acoustics, 2018). Psychoacoustic metrics

137 have been found to outperform conventional noise metrics (e.g. EPNL or SEL) in predicting noise 138 annoyance of fixed-wing aircraft (Rizzi et al., 2016; Torija et al., 2019). Recently, several authors 139 (Krishnamurthy et al., 2018; Boucher et al., 2020) have explored the potential of psychoacoustic 140 metrics for the modelling of human annoyance to rotorcraft noise, and assessed the performance of 141 each psychoacoustic metric to account for rotorcraft noise annoyance response.

142 The psychoacoustic metrics (including loudness in sone, sharpness in acum, fluctuation strength

143 in vacil, roughness in asper, impulsiveness in IU, and tonality in TU) of all sound samples were 144 calculated with ArtemiS software (HEAD acoustics GmbH). Loudness was calculated according to 145 DIN 45631/A1 (2010), which is based on Zwicker loudness model and includes a modification for

146 time varying signals. The calculation of sharpness was made according to the standard DIN 45692

147 (2009). This sharpness method does not take into account the influence of absolute loudness on the 148 sharpness perception. There are no standard methods for calculating roughness and fluctuation 149 strength. These two metrics were calculated according to the hearing model given by Sottek (1993).

150 Sottek's hearing model simulates the signal processing of human hearing and accounts for its 151 limitations to track fast temporal changes within a critical band (Boucher et al., 2020). Tonality was 152 calculated according to Aures/Terhardt tonality model (Aures, 1985b). 
154 noise annoyance. The Zwicker PA model, accounting for the relation between annoyance and 155 hearing sensations loudness $(N)$, sharpness $(S)$, fluctuation strength $(F)$ and roughness $(R)$ is given 156 by

$$
P A=N_{5}\left(1+\sqrt{w_{S}^{2}+w_{F R}^{2}}\right)
$$

158

171 findings of this research can be argued to be non-dependent of the statistical parameters used to 172 describe the psychoacoustic magnitudes. Furthermore, to avoid the transient effect of the digital 173 filters (used for the computation of the psychoacoustic metrics evaluated) at the start of the audio

sharpness, fluctuation strength and roughness metrics were used for calculating PA.

The use of $5^{\text {th }}$ percentiles in psychoacoustic analysis is a standard approach widely accepted in the literature. However, these percentile values are dependent on the recording time and the fluctuation of the psychoacoustic parameter in question. This makes that the $5^{\text {th }}$ percentile values for psychoacoustic metrics cannot be compared without appropriate background information. In this research, there is a steady sound pressure during the $10 \mathrm{~s}$ duration of each sound sample analysed. For instance, the $5^{\text {th }}$ percentile and arithmetic mean of the loudness for the rotor spacing $\mathrm{z} / \mathrm{D}=0.05$ at azimuthal angle $=12$ degrees is 98.5 and 96.4 sones respectively. Therefore, the 
174 signal analysis, the first $0.5 \mathrm{~s}$ of the sound sample was ignored in the calculation of the $5^{\text {th }}$ percentile 175 of each psychoacoustic metric.

176 As described above, the Zwicker's PA model does not include a factor for accounting for 177 the perceptual effects of tonal sounds. Di et al. (2016) derived a tonality factor (eq. 5) to develop a 178 PA model able to account for the annoyance response of sounds with strong tonal components. 179 The updated PA model developed by Di et al. (2016) $\left(P A^{\prime}\right)$ is given by

$$
P A^{\prime}=N_{5}\left(1+\sqrt{w_{S}^{2}+w_{F R}^{2}+w_{T}^{2}}\right)
$$

181

where

More (2011) developed a modified version of Zwicker PA model optimised to predict aircraft noise annoyance. The More's PA model $\left(P A_{\text {mod }}\right)$ is given by

$$
w_{T}=\frac{6.41}{N_{5}^{0.52}} T
$$

where

$$
P A_{\text {mod }}=N_{5}\left(1+\sqrt{\gamma_{0}+\gamma_{1} w_{S}^{2}+\gamma_{2} w_{F R}^{2}+\gamma_{3} w_{T}^{2}}\right)
$$
series of psychoacoustic tests. The value of these estimates for eqs. 6 and 7 , i.e. $\gamma_{0}=-0.16, \gamma_{1}=$ 11.48, $\gamma_{2}=0.84, \gamma_{3}=1.25, \gamma_{4}=0.29$ and $\gamma_{5}=5.49$, show the significant emphasis of the

191 More's PA model on sharpness and tonality. Note that $5^{\text {th }}$ percentile of Aures/Terhardt tonality was 192 used for calculating PA in Di et al.'s and More's models. 
194 is no agreement in the literature, some authors advise that the prevalence of annoyance due to 195 rotorcraft is influenced by its impulsiveness (Mestre et al., 2017). The impulsiveness (measured in

196 IU) of all sound samples were calculated using the Sottek's hearing model. This psychoacoustic

197 metric accounts for the perception caused by short and sudden changes in sound pressure level 198 (Boucher, et al., 2019). A full description of the calculation of the impulsiveness metrics and its 199 computation in the Sottek's hearing model can be found at Sottek et al. (1995) and Sottek and 200 Genuit (2005) respectively. McMullen (2014) suggested that a combination of different 201 psychoacoustic metrics, including loudness, sharpness, tonality and impulsiveness might provide an 202 accurate assessment of human response to helicopter noise. All this suggests that impulsiveness 203 might need to be considered for developing a PA model for rotorcraft noise.

204 III. RESULTS AND DISCUSSION

205 A. Directivity and spectra patterns

206 Figure 2 shows the $5^{\text {th }}$ percentile of loudness (Fig. 2C) and sharpness (Fig. 2D) as a function of 207 azimuthal angle (i.e. emission angles between 12 and 102 degrees measured relative to the rotor 208 axis), for rotor spacings $\mathrm{z} / \mathrm{D}=0.05,0.2$ and 1 and a thrust setting of $10 \mathrm{~N}$. Maximum noise emission 209 (i.e. loudness) is found at the rotor axis. Loudness decreases with azimuthal angle, reaching 210 minimum values at 82-92 degrees (Fig. 2C). The same directivity pattern is observed for all rotor 211 spacing evaluated. This is consistent with Chaitanya et al.'s (2020) previously observed optimum 212 separation distance based on overall sound power level (see Figs. 2A and 2B for equivalent sound 213 pressure level (SPL) and equivalent A-weighted SPL as a function of emission angle). Equivalent

214 SPL, equivalent A-weighted SPL and loudness are lower at rotor spacing $\mathrm{z} / \mathrm{D}=0.2$ than at rotor 215 spacings $\mathrm{z} / \mathrm{D}=0.05$ and $\mathrm{z} / \mathrm{D}=1$. 
For all rotor spacings, maximum values of sharpness are observed at azimuthal angles of 82 to 21792 degrees (Fig. 2D). Sharpness at rotor spacing z/D=0.2 is higher than sharpness at rotor spacings $218 \mathrm{z} / \mathrm{D}=0.05$ and $\mathrm{z} / \mathrm{D}=1$. Directivity patterns of loudness and sharpness metrics seem to be in line 219 with the initial hypothesis that the highest contribution to measured sounds is the noise emission of 220 potential field interaction tones. McKay et al. (2019) found that potential field interaction tones in 221 co-axial propellers have a dipole directivity with a null at 90 degrees. In this research, the dip in the 222 value of equivalent SPL, equivalent A-weighted SPL and loudness at about 82-92 degrees (as shown

223 in Figs. 2A, $2 \mathrm{~B}$ and $2 \mathrm{C}$ ) can be attributable to a decline in the noise emission of potential field 224 interaction tones. This slight shift in the dip of noise radiation from 90 degrees to 82 degrees may 225 be due to the contribution of noise sources others than potential field interaction tones, and that in 226 this research the azimuthal angles measured were related to the bottom propeller.

227 The decline in amplitude of potential field interaction tones at about 82-92 degrees also leads to an 228 important increase in the relative contribution of higher harmonics of the blade passage frequencies 229 (BPFs) and high frequency broadband noise, which is accounted for by an increase of sharpness as 230 shown in Fig. 2D. 

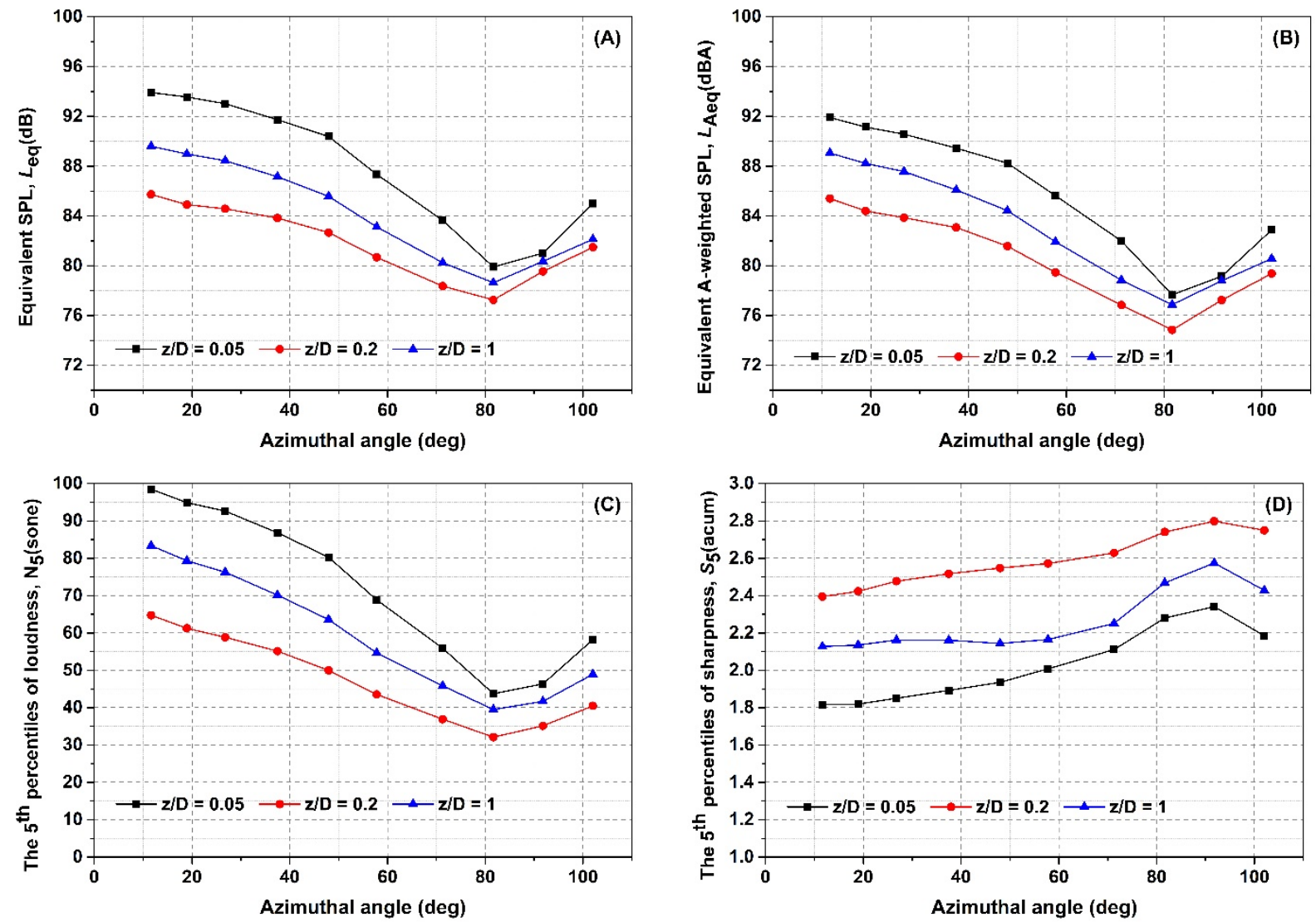

234 FIG. 2. (Color online). The equivalent SPL (A), equivalent A-weighted SPL (B), and the $5^{\text {th }}$ 235 percentiles of loudness (C) and sharpness (D) as a function of azimuthal angle, for a thrust setting of $23610 \mathrm{~N}$ and for rotor spacings $\mathrm{z} / \mathrm{D}=0.05,0.2$ and 1.

237 To continue with the investigation of the individual noise sources in the contra-rotating 238 propeller under study, a narrow band frequency analysis was conducted. Figure 3 shows the narrow 239 band frequency spectra for the rotor spacings $z / D=0.05,0.2$ and 1 for azimuthal angles 12 deg (Fig. $2403 \mathrm{~A}$ ) and $82 \mathrm{deg}$ (Fig. 3B), and a thrust setting of $10 \mathrm{~N}$. These two azimuthal angles allow the 241 comparison between the narrow band frequency spectra for high loudness (i.e. 12 deg) and high 242 sharpness (i.e. $82 \mathrm{deg}$ ). 

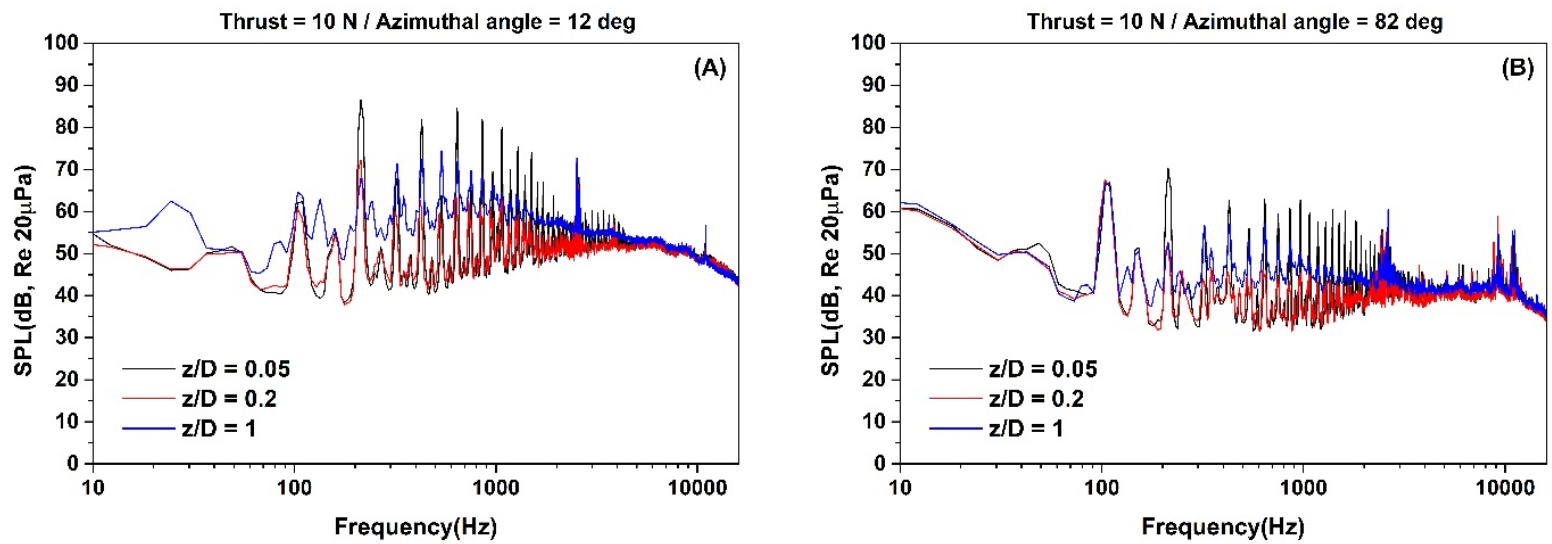

FIG. 3. (Color online). Narrow band frequency spectra for the rotor spacings $z / D=0.05,0.2$ and 1 for a thrust setting of $10 \mathrm{~N}$, and for azimuthal angles $12 \mathrm{deg}(\mathrm{A})$ and $82 \mathrm{deg}(\mathrm{B})$.

As shown in Fig. 3, the noise signatures of the contra-rotating propeller measured are dominated by tonal components distributed along the frequency spectrum (between 0.1 and $2 \mathrm{kHz}$ ). These tonal components include potential field interaction tones at frequencies that are the summation of rotor BPFs. An analysis carried out by McKay et al. (2019) and Chaitanya et al. (2020) demonstrated that interaction tones are predominantly caused by potential field interactions, and therefore, they decay rapidly with rotor spacing. This decay in amplitude of potential field interaction tones is observed by comparing frequency spectra of rotor spacings $z / D=0.05$ and 0.2 . The decrease in amplitude of potential field interaction tones as rotor spacing increases, leads to a sound signature with higher relative contribution of high frequency components (broadband and tonal components over $2 \mathrm{kHz}$ ). As the rotor spacing continues increasing, from $\mathrm{z} / \mathrm{D}=0.05$ and 0.2 to $\mathrm{z} / \mathrm{D}=1$, the contribution of broadband noise increases. This increase can be attributed to the enhanced interaction between the turbulence generated by the upper propeller tip vortex and the lower propeller as demonstrated by Chaitanya et al. (2020). significantly decays (especially for rotor spacing $\mathrm{z} / \mathrm{D}=0.2$ ), due to their dipole directivity (as described above). A decrease of about $20 \mathrm{~dB}$ is observed in the amplitude of potential field 
interaction tones at 82 degrees compared to the amplitude at 12 degrees (see Fig. 3). For the specific

264 case of rotor spacing $\mathrm{z} / \mathrm{D}=0.05$ at 82 degrees, the amplitude of potential field interaction tones is of

265 the same order of magnitude as the amplitude of BPF tones (Fig. 3B). This is due to both the dipole

266 directivity of potential field interaction tones with a null at about 90 degrees and the maximum

267 emission of BPF tones in the plane of the propeller (McKay et al., 2019). At an emission angle of 82

268 degrees there is a reduction of high frequency broadband noise (compared to 12 degrees), and a

269 series of tonal components of important magnitude are observed in the high frequency region, i.e. 2-

$27012 \mathrm{kHz}$. The precise reason for this behaviour is currently not known and more work is required to

271 understand this phenomenon.

\section{B. Psychoacoustic metrics vs. rotor spacing}

273 To investigate the optimum rotor spacing configuration for the contra-rotating system under

274 study, the value of the different psychoacoustic metrics described above in Section II.C has been

275 calculated. The value of psychoacoustics metrics ( $5^{\text {th }}$ percentile) as a function of rotor spacing at an

276 azimuthal angle of 12 and 82 degrees is shown in Fig. 4. As described above (Section III.A), at 12

277 and 82 degrees the contra-rotating system measured has the highest and lowest noise emission

278 respectively.

279 As the rotor spacing increases, the amplitude of the potential field interaction tones distributed

280 along the mid to high frequency regions decays significantly (see Fig. 3). Consequently, as shown in

281 Fig. 4A, loudness decreases with an increase in rotor spacing, reaching the lowest values at the 282 region $z / D=0.2-0.4$ at 12 degrees and $z / D=0.2-0.3$ at 82 degrees. This decay is more significant at

28312 degrees (about 30 sone reduction between rotor spacings $z / D=0.05$ and 0.2 ) where the emission

284 of potential field interaction tones is maximum, compared to 82 degrees (about 10 sone reduction

285 between rotor spacings $z / D=0.05$ and 0.2 ). At small rotor spacings, the decrease in loudness is due

286 to a reduction in the potential field interactions between the two contra-rotating propellers. This 
interaction noise is primarily tonal, and hence tonality drops significantly as rotor spacing increases

288 (see Fig. 4B). These results are in line with existing literature (McKay et al., 2019; Chaitanya et al.,

289 2020), where blade spacing optimization has been demonstrated to lead to important reductions in

290 tonal noise (Anghinolfi et al., 2016). Figure 4B shows that, at 12 degrees, there is a significant drop

291 in tonality at a rotor spacing $\mathrm{z} / \mathrm{D}=0.35$, to remain almost constant regardless rotor spacing onwards.

292 At 82 degrees, this significant drop in tonality is found at a rotor spacing $z / D=0.2$ (Fig. 4B). This

293 might be due to the directivity characteristics of potential field interactions, as described in Section

294 III.A. With higher amplitude of potential field interaction tones at emission angles about 0 degrees

295 relative to the rotor axis, a greater rotor spacing is needed at 12 degrees for tonality to drop to

296 minimum values (compared to 82 degrees). At both emission angles, 12 and 82 degrees the same

297 minimum value of tonality is observed at a rotor spacing $z / D=0.35$ (Fig. 4B).

298 Fluctuation strength accounts for the low frequency amplitude modulation consequence of the

299 closely spaced potential field interaction tones, as shown in Fig. 3. As rotor spacing increases

300 beyond $\mathrm{z} / \mathrm{D}=0.15-0.2$, potential field interactions are reduced (i.e. amplitude of interaction tones

301 decays), and consequently a significant drop in fluctuation strength is observed (Fig. 4C).

302 With increase in rotor separation distances, interaction noise between rotors increases due to

303 enhanced turbulence-propeller interactions because of unsteadiness in the tip vortex as previously

304 demonstrated by Chaitanya et al. (2020). This added turbulence-propeller interaction noise, which is

305 tonal and broadband in nature (see Fig. 3), causes an increase of loudness after rotor spacing

$306 \mathrm{z} / \mathrm{D}=0.4$ (Fig. 4A). Modulated broadband noise reaches higher roughness values than modulated

307 discrete tones, and even unmodulated broadband noise attains considerable roughness values due to

308 random envelope fluctuations (Daniel, 2008). Therefore, the increase in unsteady turbulence-

309 propeller interaction noise as rotors are moved apart might explain the gradual growth of roughness

310 shown in Fig. 4D. At 12 degrees, the highest emission of broadband noise due to unsteady 
311 turbulence-propeller interaction noise leads to a higher rate of increase in roughness with rotor 312 spacing (as observed in Fig. 4D).

313 As seen in Fig. 4E, impulsiveness significantly increases as the rotor spacing grows. This is 314 observed for both azimuthal angles of highest and lowest noise emission, although the highest 315 values of impulsiveness are at 12 degrees. As discussed by Krishnamurthy et al. (2018), 316 impulsiveness and roughness metrics are strongly linked to each other. This is observed in this 317 paper by comparing Figs. 4D and 4E. Noise caused by enhanced turbulence-propeller interactions 318 is highly impulsive, and therefore, the added turbulence-propeller interaction noise as the contra319 rotating rotors move apart from each other leads to an increase in the impulsiveness metric. This 320 suggests that the impulsiveness metric should be considered, along with roughness, to account for 321 the perceptual response to propeller-turbulence interaction noise in the development of a PA model 322 for rotorcraft noise.
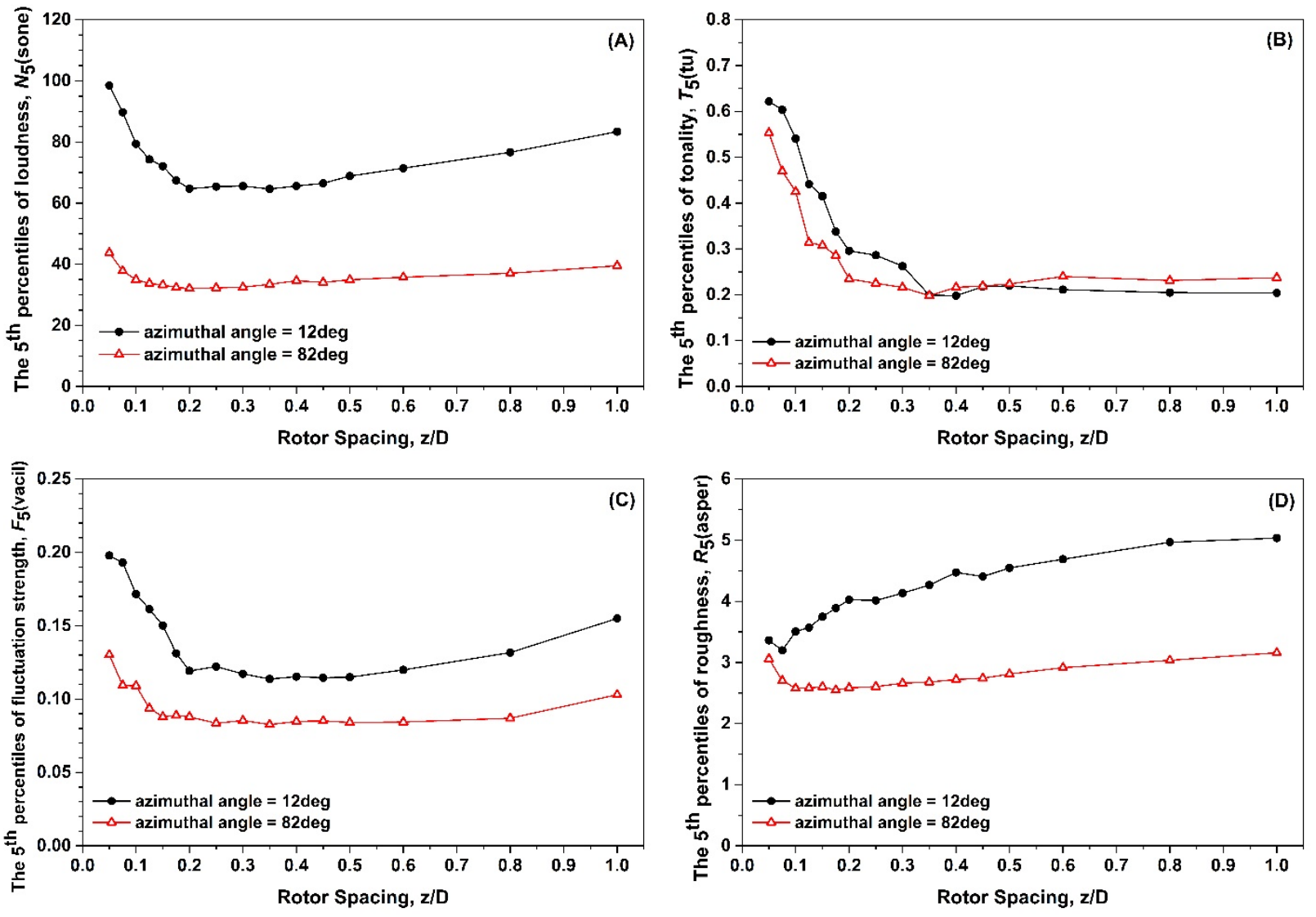

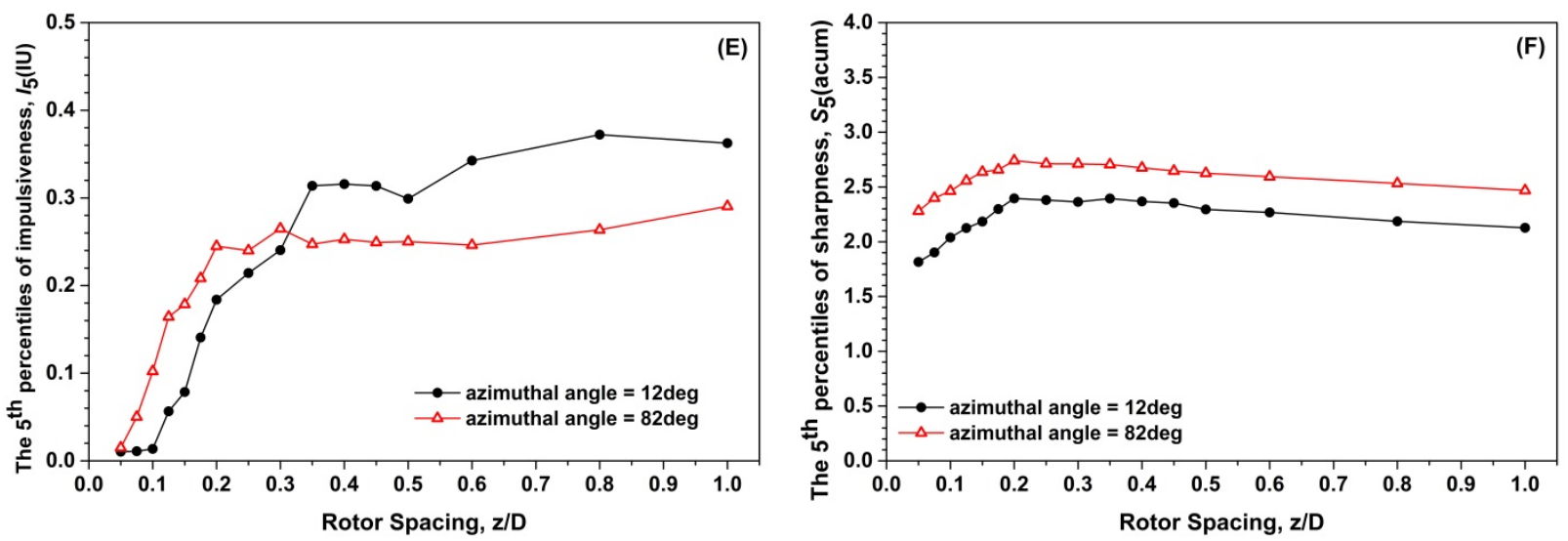

FIG. 4. (Color online). The 5th percentiles of loudness (A), tonality (B), fluctuation strength (C),

327 roughness (D), impulsiveness (E) and sharpness $(\mathrm{F})$ as a function of rotor spacing at azimuthal 328 angle12 deg and $82 \mathrm{deg}$, for a thrust setting of $10 \mathrm{~N}$.

329 At rotor spacings in the region $z / D=0.2-0.4$, the contribution of potential field interaction tones reaches a minimum. This leads to an increase in the relative contribution of high frequency tonal 331 and broadband components (i.e. shaper sounds). Therefore, at rotor spacings $\mathrm{z} / \mathrm{D}=0.2-0.4$, the 332 spectral centroid is located at a higher frequency (compared to audio signals of rotor spacings with 333 dominant potential field interaction tones), and therefore higher values of sharpness are observed 334 (Fig. 4F). The same pattern of sharpness as a function of rotor spacing is observed for both 12 and 33582 degrees, although sharpness values are higher at 82 degrees due to the lowest emission of 336 potential field interaction tones at these emission angles. Cabell et al. (2016) found important 337 emissions of high frequency tones between 3.5 and $5 \mathrm{kHz}$ for a series of multi-copters driven by 338 brushless DC motors. The noise generated by brushless DC motors is primarily due to both force 339 pulses as the magnets and armature interact and forces caused by phase changes in the motor drive 340 signal (Brackley and Pollock, 2000). Alexander at al. (2019) observed high frequency humps in a 341 series of multi-copters measured at hover configuration. Although the authors state this noise being 342 broadband in nature, its origin is still under investigation. 


\section{Models for psychoacoustic annoyance}

To identify the optimal rotor spacing configuration for the contra-rotating propeller under

345 evaluation, PA as a function of rotor spacing has been calculated according to PA models developed

346 by Zwicker and Fastl (1999), Di et al. (2016) and More (2011). As shown in Fig. 5, as expected from

347 the value of the psychoacoustic metrics analysed in section III.B, the lowest values of PA are found

348 for rotor spacing in the rage of $\mathrm{z} / \mathrm{D}=0.2-0.4$ for both 12 and 82 degrees.

349 At 12 degrees, i.e. the emission angle with the highest amplitude of potential field interaction 350 tones, three main results are observed in Fig. 5A: (i) A significant decay in PA is observed at the 351 optimal rotor spacing area, compared to rotor spacings below $\mathrm{z} / \mathrm{D}=0.2$ and above $\mathrm{z} / \mathrm{D}=0.4$. (ii) As

352 rotor interaction noise at this rotor spacing is tonal in nature (i.e. potential field interaction tones),

353 Di et al.'s PA model and especially More's PA model (both of which include a tonal factor) lead to

354 lower psychoacoustic annoyance at optimal rotor spacing than Zwicker's PA model. (iii) While

355 Zwicker's and Di et al.'s PA models give the minimum value of psychoacoustic annoyance at rotor

356 spacing $z / D=0.2$, the lowest psychoacoustic annoyance according to More's PA model is at $357 \mathrm{z} / \mathrm{D}=0.35$. This seems to be due to the higher contribution of the tonal factor in the PA model 358 developed by More (see Fig. 6).
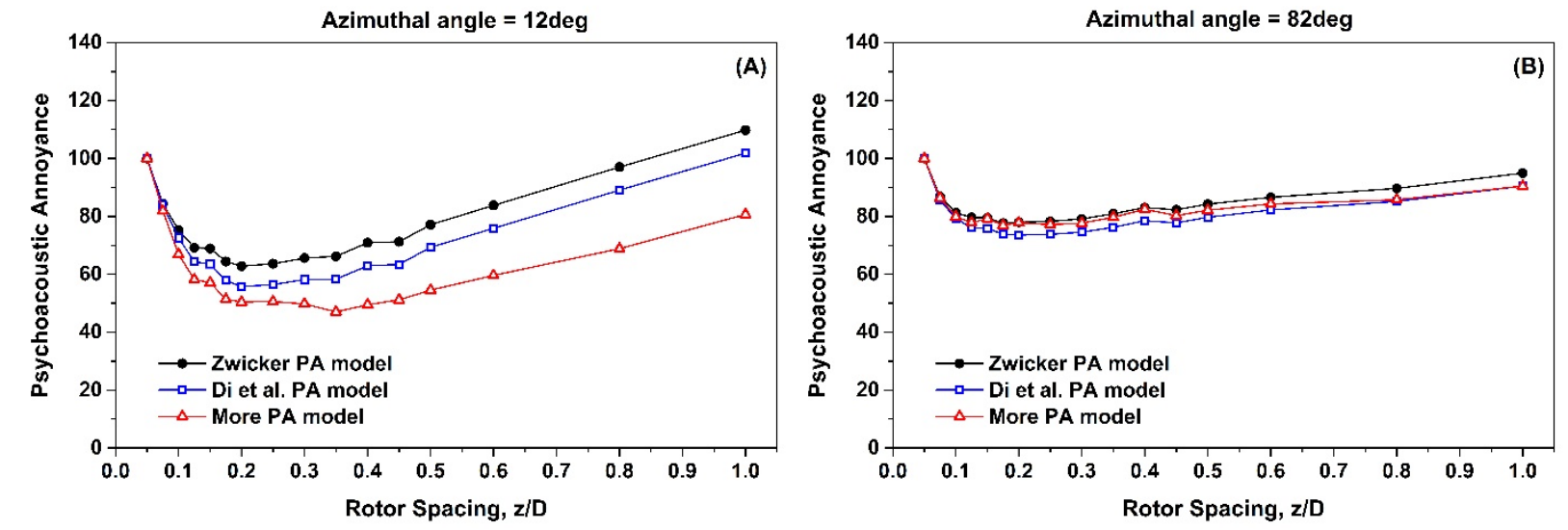

359 
360 FIG. 5. (Color online). Psychoacoustic annoyance (PA) calculated with Zwicker's, Di et al.'s and

361 More's PA models as a function of rotor spacing, for azimuthal angle $12 \mathrm{deg}(\mathrm{A})$ and $82 \mathrm{deg}$ (B) with

362 a thrust setting of $10 \mathrm{~N}$. Normalised to $\mathrm{PA}=100$ at $\mathrm{z} / \mathrm{d}=0.05$.

363 At 82 degrees, i.e. the emission angle with the lowest amplitude of potential field interaction 364 tones, it is observed that the three models implemented give similar values of PA (Fig. 5B). The PA 365 model developed by Di et al. (2016) gives the lowest values of PA among the three models used. 366 The values of PA calculated according to the model developed by More are higher than the values 367 calculated with Di et al.'s PA model for the rotor spacing range $z / D=0.15-0.6$. This seems to be 368 due to the higher contribution of the sharpness factor in the PA model developed by More (see Fig. $3694 \mathrm{~F}$ for sharpness vs. rotor spacing). At this emission angle, the range of variation of PA as a 370 function of rotor spacing is significantly more reduced than at an emission angle of 12 degrees. This 371 finding suggests that a suboptimal rotor spacing between contra-rotating propellers can lead to a 372 significant increase in PA at emission angles in line to the rotor axis. These emission angles are 373 typical for an observer on the ground interacting with a hovering contra-rotating UAV.

374 Zwicker's and Di et al.'s PA models (Zwicker and Fastl, 1999; Di et al., 2016) were derived for a 375 series of mechanical sounds, and More (2011) modified Zwicker's PA model to account for 376 characteristics of fixed-wing aircraft noise. However, none of these PA models have been optimised 377 for propeller noise, and therefore might not be able to account for the complex perceptual 378 interactions between individual noise sources (e.g. tonal components, roughness due to interactions 379 between closely spaced tones, broadband noise in high frequency region due to unsteadiness in the 380 wake, propeller-turbulence interaction noise, etc.). This might lead to important uncertainty in the 381 prediction of PA with current models available. Furthermore, in the three PA models implemented 382 in this work, loudness is included as a first order term, and the other psychoacoustic metrics are just 383 second order factors. For this reason, the calculations of PA with these psychoacoustic models are 
mainly driven by loudness, and the contribution of other psychoacoustic factors is quite reduced.

385 Sharpness has been found to be an important contributor to aircraft noise annoyance (Torija et al.,

386 2019). Sharpness, tonality and fluctuation strength were found to be important predictors of 387 annoyance for rotorcraft-like sounds (Krishnamurthy et al., 2018; Boucher et al., 2020). Roughness

388 has been found, for instance, an important factor to describing sound quality of electric motors 389 (Mosquera-Sanchez et al., 2014; Ercan, 2019). The relative contribution of psychoacoustic features 390 to annoyance for propeller noise is unknown. A process of listening tests and optimization of 391 coefficients for psychoacoustic terms in PA models, similar to the one carried out by More (2011) 392 for fixed-wing aircraft, is needed for propeller noise.

393 A recent study carried out by Gwak et al. (2020) has investigated the relationship between 394 psychoacoustic metrics and the annoyance reported for a range of hovering UAVs of varying size. 395 The authors found that the annoyance reported for medium and large drones is driven by loudness, 396 sharpness and fluctuation strength; they also found that the annoyance reported for small drones 397 cannot be explained by the three psychoacoustic metrics above, but tonality might play an important 398 role. Based on the $\beta$-coefficients of a linear regression model of the annoyance for medium and 399 large drones developed by Gwak et al. (2020, pp. 13), reported annoyance is mainly driven by 400 loudness $(\beta=0.908)$ and sharpness $(\beta=0.102)$ and fluctuation strength $(\beta=0.268)$ are second 401 order contributors. Further, the standardised $\beta$-coefficients of the linear regression model indicate 402 that an increase of 0.516 loudness units (i.e. sones) is needed to increase the annoyance in 1 unit $^{1}$, 403 while an increase of 9.902 sharpness units (i.e. acum) is needed for an increase in 1 unit of 404 annoyance. Using the results of Gwak et al. (2020), the increase in the contribution of sharpness 405 (relative lo loudness) needed in order for it to dominate the psychoacoustic annoyance calculation is 406 unrealistic. Based on this, one could argue that the optimal rotor spacing, in terms of

\footnotetext{
${ }^{1}$ Note that annoyance in Gwak et al. (2020) is assessed using a 11-point scale.
} 

general finding.
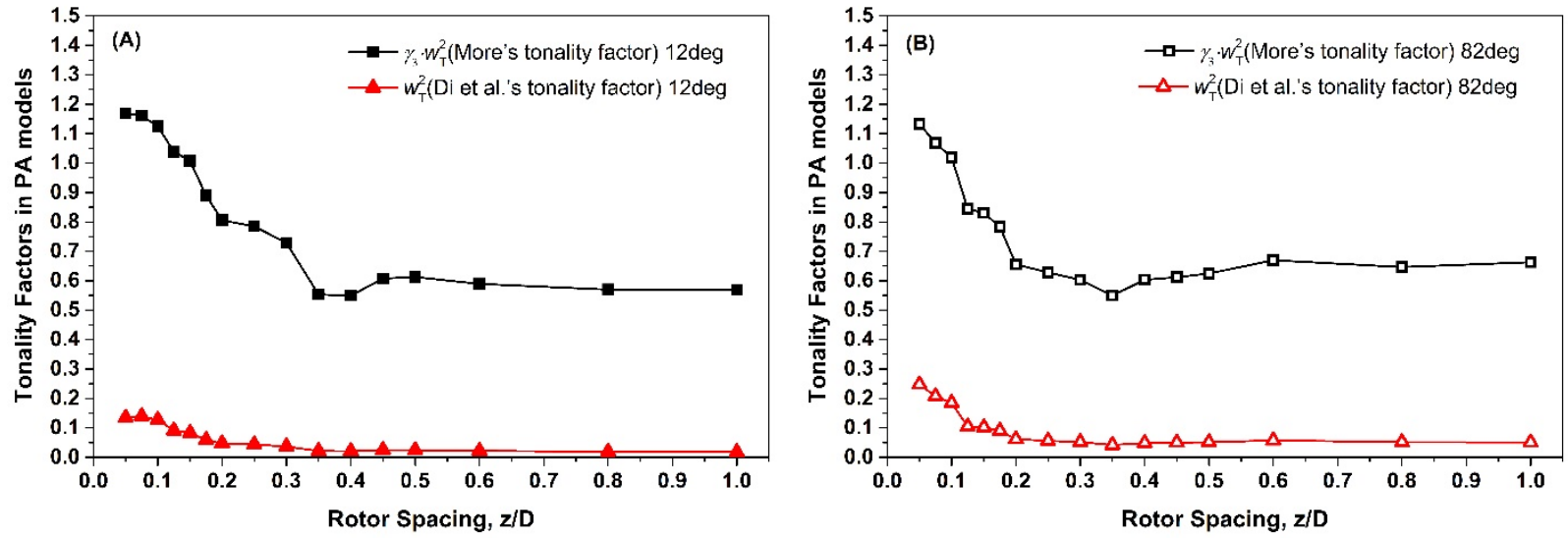

410 FIG. 6. (Color online). Di et al.'s tonality factor $\left(w_{T}^{2}\right)$ and More's tonality factor $\left(\gamma_{3} w_{T}^{2}\right)$ in PA models 411 as a function of rotor spacing, for azimuthal angle $12 \mathrm{deg}(\mathrm{A})$ and $82 \mathrm{deg}$ (B) with a thrust setting of $41210 \mathrm{~N}$

413 Although the perceived roughness and impulsiveness might be a factor due to unsteady 414 turbulence-propeller interaction noise, annoyance might be assumed to be primarily driven by 415 perceived tonality in the region of optimal rotor spacing as shown in Figs. 4B and 5 (i.e. sound is 416 eminently tonal in nature in this region due to the contribution of potential field interaction tones). 417 Several studies on a variety of noise sources, such as mechanical ventilation systems (Lee, 2016) and 418 aircraft noise (More and Davies, 2010) have suggested a combination of loudness and tonality 419 factors in multiple linear regression models as an accurate approach to predict annoyance. As seen 420 in Fig. 6, both the tonality factors derived by Di et al. (2016) and More (2011) (accounting for the 421 combined effect of loudness and tonality) suggest the optimal rotor spacing at $z / D \geq 0.35$ (note that 422 the minimum value of both tonality factors is at $\mathrm{z} / \mathrm{D}=0.35$ ). Figure 6 shows the Di et al.'s tonality 423 factor squared (eq. 5), and More's tonality factor squared (eq. 7) multiplied by $\gamma_{3}=1.25$ (to account 424 for the total contribution of tonality in More's PA model). This figure also shows that More's 
425 tonality factor emphasises more the contribution of tonality in the PA model than Di et al.'s. The

426 value of both tonality factors as a function of rotor spacing demonstrates that More's factor is more

427 sensitive to variations in tonality, and therefore would lead to higher variation in PA for the same

428 changes in tonality.

429 Future work for the development of PA models for propellers, and especially contra-rotating 430 multiple blade propellers, will need to focus on psychoacoustic features such as perceived 431 impulsiveness caused by propeller-turbulence interaction, and perceived roughness and perceived 432 tonality of multiple tone complexes. Perceived roughness of superpositioned multiple pure tones 433 (see Fig. 3) differs from perceived roughness of amplitude modulated tones, even with similar 434 modulation strengths (Terhardt, 1974; Aures, 1985a). Perakis et al. (2013) found that the modulation 435 index of an amplitude modulated tone must be lowered by $2 / 3$ to be perceived as equally rough as a 436 pair of superpositioned tones. This perceptual phenomenon should be taken into account when 437 deriving a fluctuation strength/roughness function accounting for the perceptual interaction effect 438 of closely spaced multiple tones. The perceived tonal strength of mechanical sounds containing 439 series of harmonic or inharmonic complex tones can adversely influence the perception of these 440 sounds (Lee et al., 2005). The prediction of annoyance from sounds containing multiple tone 441 complexes requires not only accounting for the tonality of the most prevailing tone and signal 442 loudness, but also the frequencies and the structure of the other tones in the noise signal (Lee and 443 Wang, 2020). Aures/Terhardt tonality model (Aures, 1985b) accounts for the presence of complex 444 tones. However, Lee at al. (2005) found that Aures/Terhardt tonality model overestimates 445 perceived tonality of complex tones. These authors modified Aures/Terhardt tonality with a factor 446 accounting for the differences in tonality perception between harmonic complexes and single tones, 447 and concluded that the perceived tonality of multiple tone complexes is a function of the pitch 448 strength of the harmonic components. Therefore, pitch perception models, such as Terhardt's 
449 virtual pitch model (Terhardt et al., 1982a;b) should be taken into account when deriving a function

450 accounting for the perceived tonality of complex tones in propeller noise.

\section{IV. CONCLUSION}

452 This paper presents the results of a psychoacoustic analysis carried out to investigate the optimal

453 distance between contra-rotating propellers to minimise noise annoyance. On the basis of 454 psychoacoustic annoyance, calculated with models available in the literature, it can be concluded that 455 the optimal rotor axial separation distance for the contra-rotating propellers under study is at a range 456 of $z / D=0.2-0.4$, instead of previously observed $z / D=0.25$ by Chaitanya et al. (2020) on the basis of 457 overall sound power level. Similar optimal rotor spacing is found for azimuthal angles of maximum 458 and minimum emission of potential field interaction tones, which are the highest contributors to the 459 contra-rotating propellers sounds measured. These results are consistent with the rotor spacing with 460 maximum aerodynamic efficiency for this contra-rotating system, measured at $\mathrm{z} / \mathrm{D}=0.3$ by 461 Chaitanya et al. (2020). Although the Aures/Terhardt tonality metric and More's and Di et al.'s 462 tonality factors suggest an optimal rotor spacing at $z / D \geq 0.35$, the psychoacoustic annoyance as 463 calculated with the three models implemented in this work significantly increases for rotor spacings 464 over $\mathrm{z} / \mathrm{D}=0.4$. Furthermore, a rotor separation over $\mathrm{z} / \mathrm{D}=0.4$ might be more impractical from a 465 construction perspective.

466 Below the optimal rotor spacing, the noise generation is dominated by potential field 467 interactions between the two contra-rotating rotors, which is consistent with previous observations 468 (McKay et al., 2019; Chaitanya et al., 2020). As the rotor spacing increases towards the optimum, 469 the magnitude of these potential field interactions lessens significantly, and therefore a decrease in 470 loudness is observed. As this source of noise is tonal in nature, tonality also drops significantly at the 471 optimum rotor spacing. This decrease in tonality, and especially loudness, lead to a minimum in 472 psychoacoustic annoyance. Fluctuation strength accounts for the slow amplitude modulation due to 
473 closely spaced potential field interaction tones, and therefore drops importantly as the amplitude of

474 these interaction tones decays.

475 With increased rotor separation distances after optimum, interaction noise between contra476 rotating rotors increases due to enhance turbulence-propeller interactions, and this leads to an

477 increase in loudness. Furthermore, as this is unsteady broadband noise in nature, roughness and

478 impulsiveness increase when rotors move apart. This suggests that the perceptual effect of 479 propeller-turbulence interaction noise could be accounted for by roughness and/or impulsiveness 480 metrics.

481 A special case takes place when calculating sharpness as a function of rotor spacing. Sharpness 482 reaches the highest values at the optimal rotor spacing region. As potential field interaction tones, 483 distributed evenly along low-to-mid frequencies, decays significantly at the optimal rotor separation 484 distance, the centroid of the spectrum moves towards the high frequency region (i.e. the relative 485 contribution of high frequency tonal and broadband noise increases). Under these conditions of 486 more dominant high frequency noise components, the values of sharpness are consequently higher.

487 The approach described in this paper, based on psychoacoustic methods available in the 488 literature, provides a more sophisticated and comprehensive analysis than traditional sound power 489 level analyses to inform the optimal design of rotating systems for lowest noise annoyance. 490 Compared to sound power level based assessments, the proposed method is able to account for the 491 key psychoacoustic features highly correlated to noise perception (e.g. tonality, roughness). 492 Appropriately accounting for the perceptual effects of key psychoacoustic factors is crucial for the 493 optimisation of designs for lower noise impact on potential exposed communities. As observed in 494 this paper, minor deviations from the optimal design (in terms of rotor spacing) of contra-rotating 495 propellers can lead to substantial increase in noise annoyance at emission angles typical for an 496 observer on the ground interacting with a hovering UAV. 
The three models implemented in this research gives the minimum psychoacoustic annoyance at

498 similar rotor spacings. Despite differences in tonality, these models are mainly driven by loudness.

499 Analysing findings of recent literature, the increase in the contribution (relative to loudness) of some

500 secondary factors (e.g. sharpness) required to become dominant for psychoacoustic annoyance

501 might be unrealistic. Based on the above, it could be argued that other psychoacoustic annoyance

502 models might also lead to the same conclusion in terms of optimum rotor spacing, and therefore,

503 the results of this paper are more general and no specific to the three annoyance models

504 implemented. However, this cannot be demonstrated without extensive testing, as it is uncertain

505 whether these psychoacoustic annoyance models provide an accurate picture of actual noise

506 perception for propeller noise (and specifically contra-rotating rotor noise). The relative

507 contribution to noise annoyance of different key psychoacoustic features in a variety of rotor noise

508 must be investigated to derive psychoacoustic annoyance models optimised for rotating systems.

509 Further work is recommended to aid the design of rotating systems for lowest noise impact: (1)

510 additional noise testing should be carried out to gather a comprehensive database with sound

511 samples of different blade geometries, thrust settings, emission angles and single vs. coaxial

512 propellers; (2) further analyses will include other psychoacoustic factors, such as impulsiveness,

513 relative approach and additional tonality models; and (3) extensive subjective testing should be

514 conducted to identify the psychoacoustic factors mainly driving rotor noise annoyance, refine or

515 compute coefficients accounting for their relative contribution to noise annoyance, and thus,

516 develop psychoacoustic annoyance models for rotor noise.

\section{$517 \quad$ ACKNOWLEGEMENTS}

518 The first author would like to acknowledge the financial support of the Royal Academy of 519 Engineering, United Kingdom (RF/201819/18/194). The authors would also like to thank Dr. 520 Mantas Brazinskas and Dr. Stephen Prior for their efforts in building this rig at the University of 
521 Southampton. Dr Zhengguang Li would like to thank the funding of Natural Science Foundation of

522 Zhejiang University of Science and Technology (No. 2019QN15).

523 REFERENCES

524 Aerospace Technology Institute (ATI) (2019). Accelerating Ambition: Technology Strategy 2019.

525 Available at: https://www.ati.org.uk/media/siybi1 mm/reduced-ati-tech-strategy.pdf (last

$526 \quad$ accessed: 07/04/2020)

527 Alexander, W.N., Whelchel, J., Intaratep, N. and Trani, A. (2019). "Predicting community noise of

528 sUAS." Proceedings of 25th AIAA/CEAS Aeroacoustics Conference, Delft, The

$529 \quad$ Netherlands.

530 Angerer, R., Erickson, R.A., and McCurdy, D.A. (1991). "Development of an annoyance model

531 based upon elementary auditory sensations for steady-state aircraft interior noise containing

532 tonal components.” NASA technical report TM 104147.

533 Anghinolfi, D., Canepa, E., Cattanei, A., and Paoluci, M. (2016). "Psychoacoustic optimization of

534 the spacing of propellers, helicopter rotors, and axial fans." Journal of Propulsion and

$535 \quad$ Power, 32(6), 1422-1432.

536 Aures, W. (1985a). "Ein Berechnungsverfahren der Rauhigkeit (A Calculation Method for 537 Roughness).” Acustica 58, 268-280.

538 Aures, W. (1985b). "Berechnungsverfahren für den sensorischen wohlklang beliebiger schallsignale"

539 (“A procedure for calculating sensory pleasantness of various sounds.”). Acta Acustica $540 \quad$ united with Acustica, 59(2):130-141, 1985.

541 Barbot, B., Lavandier, C., Cheminee, P. (2008). "Perceptual representation of aircraft sounds." $542 \quad$ Applied Acoustics, 69, 1003-1016. 
543 Berckmans, D., Janssens, K., Van der Auweraer, H., Sas, P., and Desmet, W. (2008). “Model-based 544 synthesis of aircraft noise to quantify human perception of sound quality and annoyance." Journal of Sound and Vibration, 311, 1175-1195.

546 Boucher, M., Krishnamurthy, S., Christian, A., and Rizzi, S.A. 2020. "Sound quality metric indicators of rotorcraft noise annoyance using multilevel regression analysis”. Proc. Mtgs. Acoust. 36,

Brackley, M., and Pollock, C. (2000). "Analysis and reduction of acoustic noise from a brushless dc drive.” IEEE Transactions on Industry Applications, 36(3), 772-777.

Brazinskas, M. (2019). “An empirical investigation into interference effects of small-scale rotors for use in vtol unmanned aircraft". PhD thesis, University of Southampton.

553 Cabell, R., Grosveld, F., and McSwain, R. (2016). "Measured noise from small unmanned aerial 554 vehicles." Proceedings of NOISE-CON 2016, Vol. 252, Institute of Noise Control Engineering, Providence, RI, USA.

556 Chaitanya, P., Joseph, P., Prior, S. D., Parry, A. B. (2020). "On the optimum separation distance for 557 minimum noise of contra-rotating propellers.” Journal of Fluid Mechanics (Under review). 
566 Di, G-Q., Chen, X-W., Song, K., Zhou, B., and Pei, C-M. (2016). 'Improvement of Zwicker's 567 psychoacoustic annoyance model aiming at tonal noises.” Applied Acoustics 105:164-170.

568 DIN 45631/A1-2010. "Calculation of loudness level and loudness from the sound spectrum 569 Zwicker method - Amendment 1: Calculation of the loudness of time-variant sound."

570 DIN 45692-2009. "Measurement technique for the simulation of the auditory sensation of 571 sharpness."

572 Ercan, A. M. (2019). "Sound quality of small electric motors." Proceedings of InterNoise 2019, $573 \quad$ Madrid, Spain.

574 Gwak, D.Y., Han, D., and Lee, S. (2020). "Sound quality factors influencing annoyance from 575 hovering UAV.” Journal of Sound and Vibration, 115651.

576 HEAD Acoustics (2018). "Loudness and sharpness calculation.” Technical Report Application Note $577-02 / 18$

578 Heff, G.E. (1990). "Experimental performance and acoustic investigation of modern, 579 counterrotating blade concepts.” NASA Contractor Report 185158.

580 Krishnamurthy, S.,Christian, A., and Rizzi, S. (2018). "Psychoacoustic test to determine sound 581 quality metric indicators of rotorcraft noise annoyance.” Proceedings of Inter-Noise 2018 582 Impact of Noise Control Engineering, Chicago, USA.

583 Krishnamurthy, S., Rizzi, S.A., Boyd Jr., D.D., and Aumann, A.R. (2018). “Auralization of rotorcraft 584 585

586

587 periodic flyover noise from design predictions.” Proceedings of the AHS International 74th Annual Forum \& Technology Display, May 14-17, 2018, Phoenix, Arizona, USA.

Lee, K.H., Davies, P., and Surprenant, A.M. (2005). "Tonal strength of harmonic complex tones in machinery noise.” Journal of the Acoustical Society of America 118, 1921.

588 Lee, J. (2016). "The effects of tones in noise on human annoyance and performance." $\mathrm{PhD}$ 589 dissertation, University of Nebraska. 
Lee, J., and Wang, L.M. (2020). "Investigating multidimensional characteristics of noise signals with tones from building mechanical systems and their effects on annoyance." Journal of the Acoustical Society of America 147, 108-124.

593

Lyon, R.H. (2003). "Product sound quality - from perception to design.” Sound and Vibration, 594 $37(3), 18-23$.

McMullen, A.L. (2014). “Assessment of noise metrics for application to rotorcraft.” Master's Thesis, Purdue University.

597 Magliozzi, B., Hanson, D.B., and Amiet, R.K. (1991). "Propeller and propfan noise." In Harvey H. Hubbard, editor, Aeroacoustics of Flight Vehicles: Theory and Practice: Volume 1: Noise Sources, number NASA RP-1258, pages 1-64.

600 Marte, J. E. \& Kurtz, D. W. (1970). "A review of aerodynamic noise from propellers, rotors, and lift 601 fans.” NASA Technical Report NASA-32-7462.

602 McKay, R.S., Kingan, M.J. and Go, R. 2019. "Experimental investigation of contra-rotating multi603 rotor UAV propeller noise.” Proceedings of ACOUSTICS 2019, Cape Schanck, Victoria, $604 \quad$ Australia.

605 Mestre, V., Fidell, S., Horonjeff, R.D., Schomer, P., Hastings, A., Tabachnick, B.G., and Schmitz, 606 F.A. (2017). “Assessing Community Annoyance of Helicopter Noise." Washington, DC: 607 The National Academies Press. Available at: https://doi.org/10.17226/24948.

608 More, S, and Davies, P. (2010). "Human Responses to the Tonalness of Aircraft Noise." Noise $609 \quad$ Control Engineering Journal 58.4, 420-440.

610 More, S. (2011). “Aircraft noise metrics and characteristics.” PhD Thesis, Purdue University.

611 Mosquera-Sánchez J.A., Villalba, J., Janssens, K., and de Oliveira, L.P.R. (2014). “A multi objective 612 sound quality optimization of electric motor noise in hybrid vehicles." Proceedings of 
International Conference on Noise and Vibration Engineering (ISMA 2014), Leuven, Begium.

615 Perakis, G.J., Flindell, I.H., and Self, R.H. (2013). "Toward Roughness as an additional metric for 616 aircraft noise containing multiple tones.” Acta Acustica United with Acustica 99, 828-835.

617 Rizzi, S.A. (2016). "Toward reduced aircraft community noise impact via a perception-influenced design approach.” Proceedings of InterNoise 2016, Hamburg, Germany.

619 Rizzi, SA., Burley, C.L., and Thomas, R.H. (2016). "Auralization of NASA N+2 Aircraft Concepts 620 from System Noise Predictions." Proceedings of the 22nd AIAA/CEAS Aeroacoustics Conference. Lyon, France.

622 Sottek, R. (1993). "Modelle zur Signalverarbeitung im menschlichen Gehör" ("Models for signal 623 processing in human hearing.”). PhD thesis, RWTH Aachen.

624 Sottek, R., Vranken, P., and Busch, G. (1995). “A model for calculating impulsiveness” (“Ein Modell 625 zrur Berechnung der Impulshaltigkeit”). Proceedings of DAGA 95 (German Acoustical 626 Society Meeting), Saarbrücken, Germany.

627 Sottek, R., and Genuit, K. (2005). "Models of signal processing in human hearing”. International 628 Journal of Electronics and Communications (AEU) 59, 157-165.

629 Stract, W. C., Knip, G., Weisbrich, A. L., Godston, J. and Bradley, E. (1981). "Technology and 630 Benefits of Aircraft Counter Rotation Propellers.” NASA Technical Memorandum 82983. Available at: https://ntrs.nasa.gov/archive/nasa/casi.ntrs.nasa.gov/19830002859.pdf (last accessed: 07/04/2020)

633 Terhardt, E. (1974). "On the perception of periodic sound fluctuations (roughness)." Acustica 30, 634 548-560. 
635 Terhardt, E., Stoll, G., and Seewann, M. (1982a). "Pitch of complex signals according to virtual636 pitch theory: Tests, examples, and predictions." Journal of the Acoustical Society of $637 \quad$ America 71, 671-678.

638 Terhardt, E., Stoll, G., and Seewann, M. (1982b). "Algorithm for extraction of pitch and pitch 639 salience from complex tonal signals." Journal of the Acoustical Society of America 71, 679$640 \quad 688$

641 Tinney, C.E. and Sirohi, J. (2018). "Multirotor drone noise at static thrust." AIAA Journal, 56(7), $642 \quad 2816-2826$.

643 Torija, A.J., Roberts, S., Woodward, R., Flindell, I.H., McKenzie, A.R., and Self, R.H. (2019). "On 644 the assessment of subjective response to tonal content of contemporary aircraft noise." $645 \quad$ Applied Acoustics, 146, 190-203.

646 Torija, A.J., Self, R.H., and Lawrence, J.L.T. (2019). "Psychoacoustic characterisation of a small 647 fixed-pitch quadcopter.” In: Proceedings of InterNoise 2019, Madrid, Spain.

648 White, K., Bronkhorst, A.W., and Meeter, M. (2017). "Annoyance by transportation noise: the 649 effects of source identity and tonal components." Journal of the Acoustical Society of 650 America, 141, 3137-3144.

651 Zwicker, E. and Fastl, H. (1999). "Psychoacoustics - facts and models." Berlin: Springer-Verlag. 